\title{
THE USE OF PORCELAIN AS A FILLING MATERIAL.
}

\author{
By J. E. Argue, D. D. S., Tacoma, Washington.
}

(Read before the Washington State Dental Association June, 1918.)

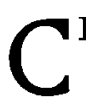

ERTAIN definite principles should be applied in making a porcelain inlay operation that will standardize its use, thus increasing its permanency to the extent that it will unquestionably hold the high place in dentistry that it should. From an esthetic standpoint, in exposed surfaces of the teeth, when it is properly placed, it gives to the profession a field where the greatest artistic results can be obtained. We can feel duly grateful to some of the most conscientious men who have given us some very valuable contributions in the wai of methods and technic in this field which have done so much for the development of porcelain.

However, in the past there has also been a number of most valuable principles overlooked, which has jeopardized its success to the extent that the greater part of the profession had for a time, lost all confidence in porcelain. A few of these errors which have been the cause of failure, are the disregard of the edge strength of porcelain; some claiming it so much superior to gold or any other filling material, advocating it being placed in any and all surfaces, and where the occlusion came directly on it, and fracture at the cavo surface angles was the result, which caused the failure of the operations to an alarming percentage.

Also, great rounded cavity outlines were made by some operators, which lacked extension at the proper angles, mutilating and weakening tooth structure, faulty adaptation and overhang at the cavo surface angles, which caused irritation and disease of the soft tissues, and thus invited recurrence of caries.

So it was, that many of the important principles were overlooked; but as development has gone on in other branches of dentistry, porcelain has not stood still, it receiving the recognition of applying certain required principles. I can enthusiastically say, that porcelain is gradually and forcibly coming into its own as a permanent filling material. lain.

So much for the past history of porce-

What I wish to outline, is in the way of applying definite and practical principles that should apply to any and all filling operations, to make them consistent with the modern dentistry of today.

Porcelain stands very high and also very low in some of the physical properties for the requisites of a filling material, which require the field for its use to be narrowed to where the demand for its greatest physical properties can be fulfilled and at the same time eliminate causes of failure due to its low physical properties. Its greatest physical property is that of harmony with the tooth color, which gives it such a high place from an esthetic standpoint. I would consider that the esthetic be made the principal basis for its use. So, therefore, its field should be confined to surfaces of the teeth that are exposed while the lips are drawn back.

Its lowest physical property is that of edge strength, which varies according to the fusing point of the porcelain, the higher fusing bodies having the greatest edge strength. In bulk, with no frail 
edges exposed, as when used in building up the Land porcelain crown, the higher fusing bodies afford the greatest resistance to attrition and sustaining power against the stress of mastication, but when made into inlays in the usual manner, thin edges are formed at the point where it approximates the cavity wall with the tooth surface, known as the cavo surface angles. These thin edges of porcelain, when subjected to the stress of occlusion, will fracture, which is due to the lack of edge strength. In surfaces of the teeth, where it has been selected for a filling operation from an esthetic standpoint where the cavo surface angles are subjected to the stress of occlusion, it should be relieved from this stress by using a combination of gold at the cavo surface angles.

In all the other physical requirements, porcelain stands very high, with exception of the difficulty of adaptation to the cavity wall, but this even can be accomplished by using a careful technic.

The porcelain inlay can then be divided into two classes: Those for surfaces of teeth where the stress of occlusion is practically nothing, (on the cavo surface angles where porcelain can be used alone, known as a porcelain inlay operation) and those in surfaces of the teeth where the stress of occlusion comes somewhat directly on the cavo surface angles requiring the combination of gold with the porcelain, known as a combination porcelain and gold inlay operation.

The field for porcelain inlay operations is limited to the proximal surfaces of the upper and lower incisors and cuspids; it should not be used where the incisal angle is involved.

Also to the labio-gingival surfaces of the upper incisors, cuspids and sometimes the first and second bicuspids, depending on how much these surfaces are exposed, while the lips are drawn back. The labio-gingival surfaces of the lower incisors and cuspids are, as a rule, covered much more by the lower lip, while smiling, than the upper corresponding teeth by the upper lip. It depends entirely on how much these surfaces are exposed in the lower incisors and cuspids; very rarely would it be recommendable to use porcelain inlays in the bucco-gingival surfaces of the lower first and second bicuspids.

The field for the combination porcelain and gold is in the proximo incisal surfaces of the upper and lower incisors and cuspids, and sometimes the mesioocclusal surface of the upper first bicuspid, depending on the extent of the caries, the alignment of the teeth and how much this surface is exposed while the lips are drawn back.

In the general consideration for the application of porcelain as a filling material, the operator should develop a keen observation of conditions that exist in each case, taking into account, in the broadest measure, the general principles that govern the making of any filling operation and applying them in a scientific manner to the material used.

These principles are, namely:

The esthetic.

Extent of the caries.

Stress of occlusion.

Planes of the occlusion.

The area of comparative immunity.

The access to placing the operation.

The alignment of the teeth.

After the case has been properly studied, the operation is then begun. The general procedure I have divided for convenience of study, into seven steps.

1st. Cleaning of the surface of the teeth.

2nd. Separation.

3rd. Cavity preparation.

4th. Formation by swaging and burnishing the matrix.

5th. Selection and building in colors.

6th. Building of contour and tooth form.

7th. Removal of the platinum matrix and cementation.

1st. The teeth are cleaned by remov- 
ing the discolorations on their surfaces with flour of pumice and a rubber cup on a mandrel which is in the engine. The mouth is then washed with some warm water in which a few drops of oil cassia has been placed, flushing thoroly around the teeth with the water syringe. This affords the operator a clean field, and an opportunity to study the correct color of the teeth.

2nd. Separation. The use of separation is for the purpose of giving convenience to the forming of the matrix and access so that the cavity can be prepared with definite walls. With ample space between the cavity walls and the proximating tooth, the completed inlay can be set to place without difficulty. In the proximal surface of the incisors and cuspids, not including the incisal angle, is the principal place that separation is necessary in placing inlay operations. If separation has not been previously made by gutta-percha or rubber, the separation must be made direct by the use of the separator.

3rd. Procedure in cavity preparation does not differ in general procedure from that for the preparation of any permanent filling material which consists of:

Outline form, Retentive form, Resistance form, Convenience form, Marginal form, Removal of Decay and Making the toilet of the cavity.

The outline form is of particular importance. In making porcelain operations, in view of the fact that when it is carried out along the proper lines it aids greatly in making the operation more imperceptible to the eye, and therefore a more esthetic result is obtained. But the greatest reason of all is to extend the surfaces of the operation to an immune area, and as well conserve tooth substance to a minimum.

On the proximal surfaces of the incisors and cuspids, but not for cavities including the angle, the outline is as follows: The gingival outline should extend just below the gingiva, thus fol- lowing its curvative, labiolingually, but in cases where recession of the gingival tissue has taken place, the margin is not carried under the gingiva.

The labial and lingual outline should be well beyond the contact point, it depending entirely on the approach of the proximating surface, carrying it to an arei where it would be kept clean by the sweep of the excursions of the food, the tongue and lips, to a line running gingivoincisally on the labial and lingual surfaces in the general direction of the lobes of the teeth, meeting the gingival outline nearly at an acute angle, but it must be slightly rounded for porcelain, to give more edge strength to the inlay at that point, as is shown in figure 1 .

Incisally it should meet the incisal outline at a short but graceful curve.

The incisal outline should be nearly at right angles to the labial and lingual outlines and beyond the point of contact incisally, as is shown in figure 1.

The greatest care should be taken, in making this outline, so as not to weaken the incisal angle.

Outline for Cavity in the Proximal Surfaces of the Incisors and Cuspids including the Angle.

The gingival outline would be the same as already spoken of. The labial and lingual outline would differ in that the labial outline would extend on a straight line to within one-half to one millimeter, and on the lingual surface, to one and one-half to two millimeters of the incisal surface, as shown in figures 3 and 4.

The incisal outline continuing at right angles, or nearly so, mesially or distally, as the case may be, to a point just beyond the middle of the middle lobe of the tooth, extending them to the incisal surface at right angles, with the angle slightly rounded.

Combination porcelain and gold is recommended in this surface. In the lower incisors, the outline form differs on account of difference of the occlusion; the labioincisal surface is extended from 
one to one and a half millimeters giving that added thickness of gold where the greatest occluding stress comes direct.

The outline for the gingival surfaces should extend just beneath the gingiva, when the gingival tissue is normal, otherwise not, mesially and distally, to a point just within the mesiolabial and distolabial angles along straight lines conform-

LABIAI.

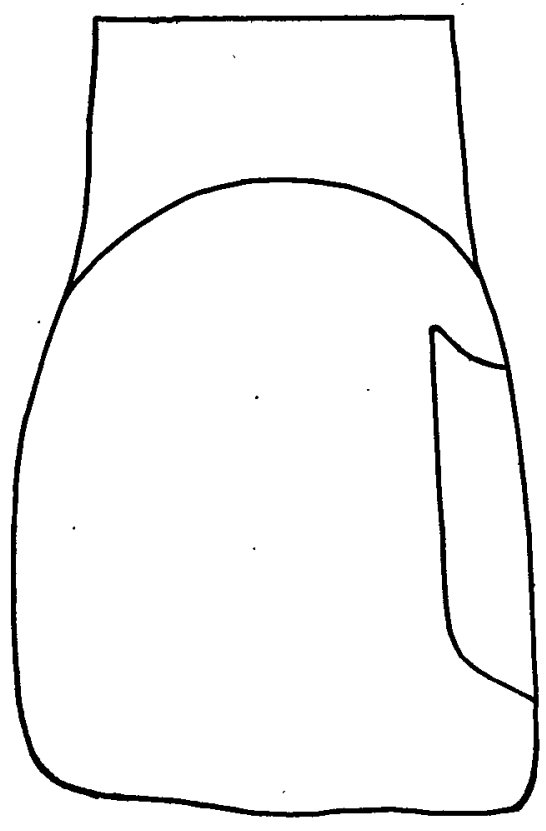

Figure 1.

ing to the general direction of the lobes, gingivoincisally, and the incisal outline should vary according to the form of the labial surface, as it must be carried to a point incisally about to its greatest convexity, as illustrated in figure 8 , where it can be reached by the sweep of the lips and the excursions of the food keeping it clean and free from further attacks of caries. These outlines should all meet at slightly rounded angles rather than acute ones for the reason that it gives greater edge strength to the inlay at this point, as shown in figure 2.
The retentive form for porcelain inlay is made by forming definite walls meeting at definite sharp angles, and as nearly at right angles to each other as the access will permit.

In the proximoincisal surfaces, where the combination porcelain and gold inlays are used, in addition to deffäit walls and angles, a pit is sunk in the

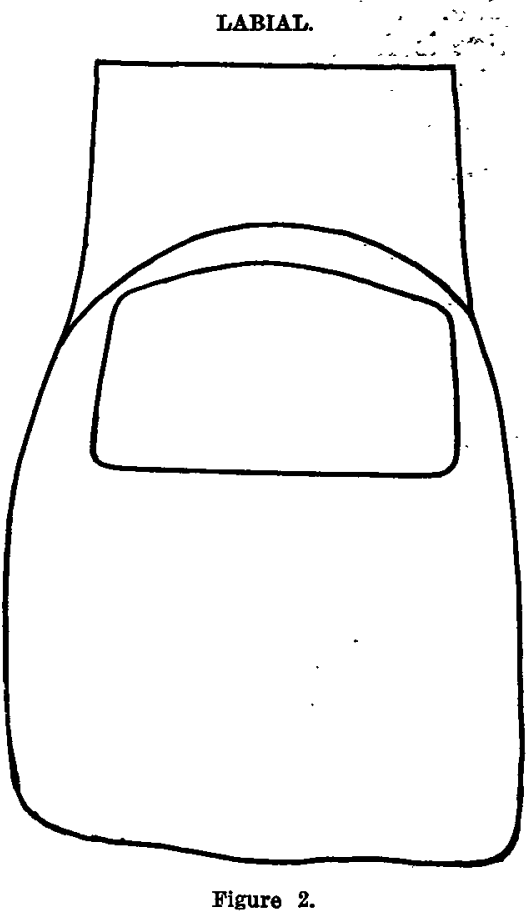

gingival wall, lingually, and in the pulpal wall, mesially or distally, as the case make be, with a 56 fissure bur, to a depth of one-half of the length of the leaves of the bur; this reproduces two spurs on the gold portion of the inlay, as shown in illustration 4, thus giving a great additional anchorage to the inlay and when the porcelain or labial half of the inlay is cemented to place first, and then the gold or the linguoincisal part of the inlay is cemented to place; the gold covers the incisal part of the porcelain, locking it into place giving the 
greatest retentive form for the porcelain as is illustrated in 3 and 7.

Resistance form to stress is accomplished by making broad flat gingival and pulpal walls in all cases, these meeting the axial wall at acute right angles.

Convenience Forms. In cases where perations are made in cavities of initial decay in proximal surfaces, no more of

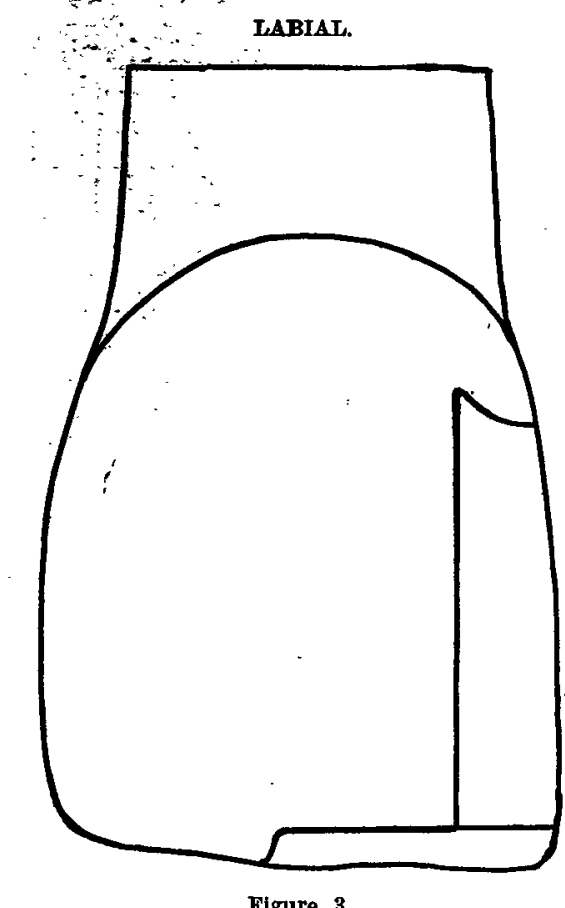

the tooth should be cut away than would be permitted to establish the outline into immune area. Convenience should be made by beveling the labial wall labially and by separation, thus making the cavity more accessible in placing the completed inlay. In case of extreme caries in these surfaces, the lingual wall is usually weakened greatly, and it can be cut away as shown in figure 5 . This gives all the convenience necessary to place the inlay from the lingual surface.

Marginal form consists of the management of the enamel rods. A knowl- edge of the histological structure of the enamel is necessary to understand the direction they run on the different surfaces of the teeth.

For porcelain inlays, the marginal form differs from that cut for gold, in the fact that it is not subjected to mallet force in placing the operations and does not require th, beveling, therefore the

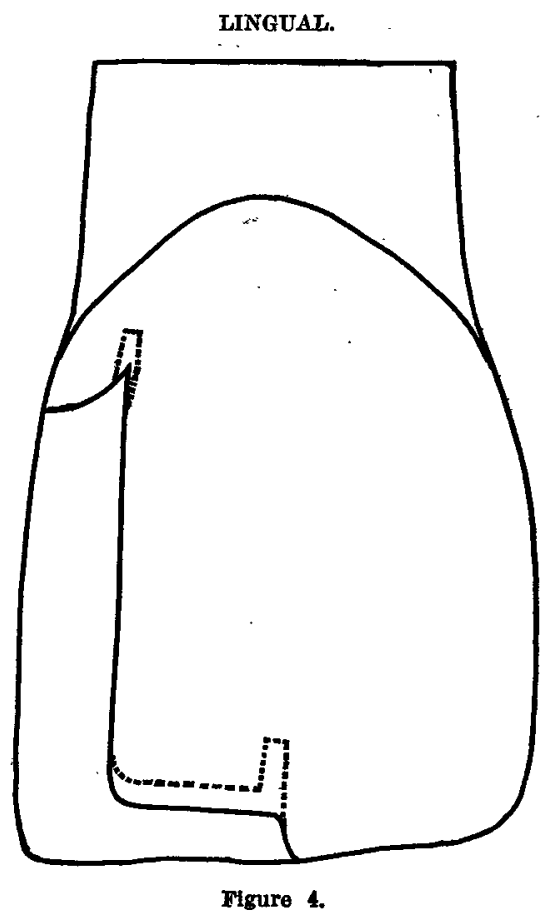

enamel is trimmed on a line with the long axis of the rods, thus giving the porcelain an equal advantage to stand the stress, but where the rods are short, as on the gingival cavo-surface angle, it is a different problem. There, the rods are more liable to chip or fall out in forming the matrix and they should be given a bevel. Here, the porcelain is not subjected to stress and if the thin edge of porcelain that covers this bevel, is carefully managed, in removing the matrix and cementing, it would be the only precaution to be taken. In the com- 
bination of porcelain and gold inlay, the enamel is given a bevel for the gold part of the inlay.

Removal of the Decay. When the preparation has been made complete in outline and the walls shaped to form, all the discolored and stained dentin should be carefully removed by using spoon excavators, as any stained dentin would reflect thru the teeth and there

LINGUAL.

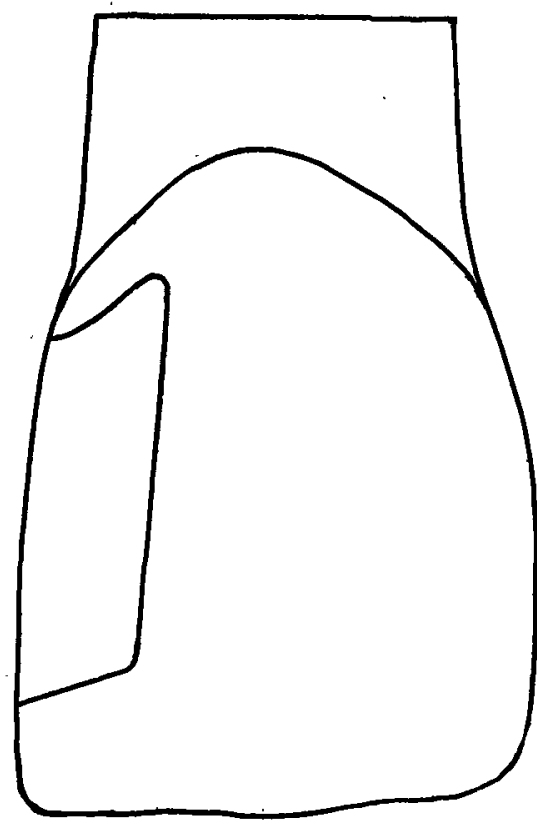

Figure 5.

would be a possibility of an off colored shade.

The toilet of the cavity is made, but not until the inlay is completed and ready to set. The walls and angles should be gone over with a No. 5 hoe excavator; they should then be dehydrated with alcohol and dried with warm air.

4th. Formation of the Platinum $M a-$ trix. Platinum foil, rolled to $1-1000$ inch thickness, is used. It can be annealed by placing it in the furnace and heating to about 2100 degrees for fifteen to twenty minutes.
There are two methods of forming the matrix for a porcelain inlay, the direct and the indirect. Of the two methods, advantages are claimed for both, and I would say that when a careful technic is followed out, you can obtain results with the use of either. However, I am partial to the direct methods for the following reasons: That the operations can be made at one sitting; the advant-

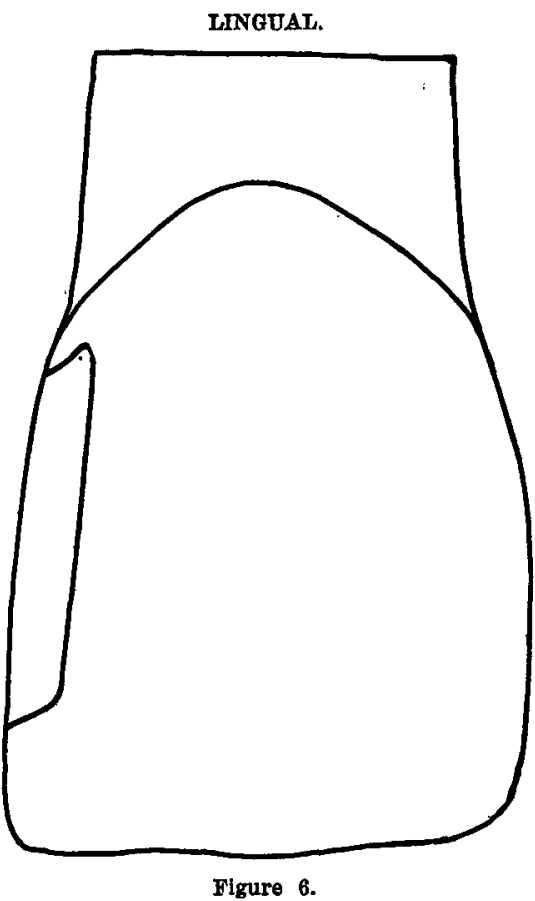

age to study the color and tooth form; also, I claim that you can get more accurate adaptation of the porcelain at the cavo-surface angles which enables the operator to turn out an inlay with less overhang at that point.

In proximal surfaces, the matrix is cut one millimeter larger than the outline of the cavity at all points. The piece of platinum is set to place with a pair of foil pliers and a burnisher is first used to carry it over the cavo-surface angles. Then a piece of spunk, that will fill the cavity, is moistened and 
pressed against the platinum, forcing it into the cavity with a ball burnisher. A flat ended orangewood stick is then used and with the aid of the mallet, this spunk is forced into the cavity which carries the matrix against the walls and down into the angles. The spunk is then removed; then a strip of medium rubber dam is passed around the tooth over the platinum and it is held tightly

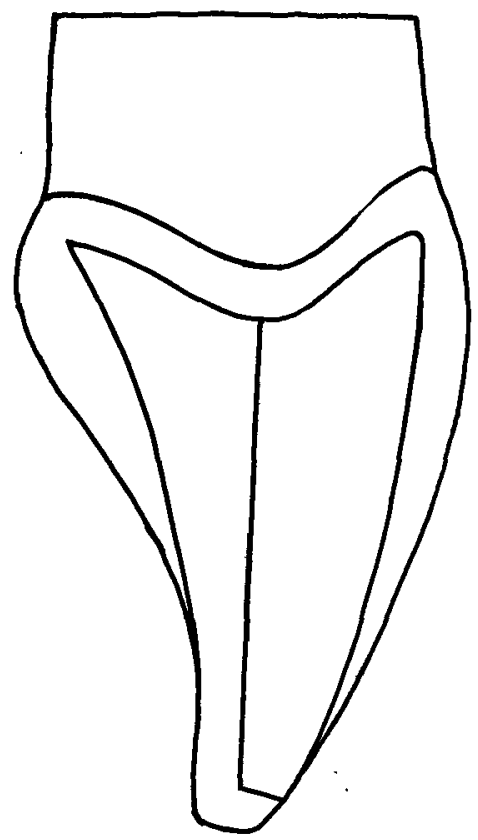

Figure 7 .

while the burnishing is completed, first by using larger ball burnisher; then a smaller sized one is used, working it well down into the cavity and with a rounded point No. 6 explorer; the final burnish is made down into the angles, which gives a clean angular outline to the matrix reproducing the gingivoaxial, linguoaxial, labioaxial and incisoaxial, angles. The matrix is then removed and trimmed, leaving about one-half millimeter overlap at all points except at the linguocavo surface angle. At that place it should be trimmed so that it barely covers the angle. This enables the operator to remove the matrix from the labial surface with much greater ease. It is then replaced in the cavity, the rubber band passed around it and held firmly while it is given a very careful reburnishing at all points. Care should then be taken in its removal from the cavity, so as not to change its form in the least, and if the proper care is used

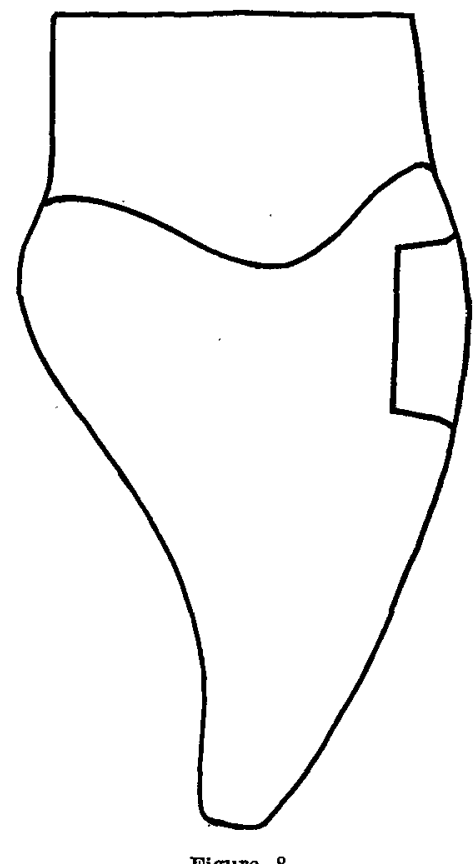

Figure 8.

in baking the porcelain no change will take place whatever in the matrix. After the first bake, it is returned again to the cavity and a rubber passed around it and a careful burnish is given the cavosurface angles.

In labial surface cavities a somewhat different technic must be employed, as here the placing of the matrix into the cavity is more difficult, and I wish to give you a simple technic which overcomes this diffculty completely. An orangewood stick is shaped one-fourth of a millimeter smaller than the cavity 
outline; this allows room for the matrix between the orangewood and the cavity wall when the matrix is carried to place.

A piece of platinum foil is then cut two millimeters larger than the cavity outline; this is folded over the end of the orangewood. This is then introduced and with the mallet is driven down into the full depth of the cavity; the orangewood is held firmly with the left hand, while with a flat burnisher, the platinum that protrudes, is unfolded and burnished over the labial surface.

The orangewood is removed and wet spunk placed; mallet force is then applied on the orangewood which swages the spunk, thus forcing the matrix against all walls of the cavity, also, over the cavo-Eurface angles.

The matrix is removed and trimmed, leaving one-half to one millimeter of an overhang. It is again replaced in the cavity, swaged to place with the spunk, using the orangewood; the spunk is then removed and a very careful final burnish into all the angles is given with a No. 6 explorer burnisher; at this point gum camphor is placed into the matrix, then by using the orangewood and mallet on the gum camphor, the final swage is given the matrix. This then is removed with a No. 6 explorer and wax is flowed into the matrix and chilled; then the matrix is removed, the wax allowing its removal without changing form. This wax is then burned out over a flame. In forming the matrix for the porcelain, in the combination porcelain and gold inlay, the same technic is followed out as for the cavities in proximal surfaces, extending the matrix over the area of the cavity that you wish to have the porcelain placed.

5th. Building in Shades. Much can be written on the shading and building shades of porcelain. However, I would suggest that we apply the simplest method consistent with a technic to obtain the proper result.

The different shade guides have am- ple shades of varying colors so that very little mixing of colors is necessary. The tooth surface is at all times bathed in the saliva; therefore the teeth should be kept moist in making the study of the colors, for the varying blends stand out in exactly the same proportion as under the same conditions that they should be reproduced. Selecting the shades for a porcelain inlay is a problem that requires the development of a keen eye with a delicate perception of the varying colors that blend from the gingival to the incisal in a tooth, and its success in reproducing them, depends on how correctly we can read them. If you make a study of the teeth in the mouth, it is found that this graduation of color varies greatly in different cases and differs somewhat in the teeth of the same person. This graduation of color is found to be, colors that lie principally within the dentin, so arranged to give abruptness or delicacy of blend into one another that reflects thru the translucent plates of the enamel.

After the study of the tooth's color is made, select a color a degree lighter in hue; always take into account the thickness the porcelain will be built at any given point of the inlay, for the reason that the thicker the body of any given color is built, the denser the color, and on the other hand the thinner the body, the paler or lighter the color will be the result. It may be necessary to use two, three and sometimes four variation of shades and in some cases distinctly different colors. This is governed by the extent of the surface involved, gingivoincisally, and the degree of the blend necessary for the case. If the blendings end abruptly into one another, the technic should be as follows:

The porcelain is worked as dry as possible, into first, the gingival part of the matrix and then add the remaining colors on this, toward the incisal, with the number of colors necessary at the given areas, till the incisal part of the 
matrix is reached, not allowing them to run together, until they are all placed. Then by gently vibrating till they barely run together, this leaves the colors with an abrupt ending into one another.

For a delicate blend, fewer colors should be used, working the body more moist so that when the varying colors are placed and the matrix vibrated, they will run further into one another, thus giving a more constant and delicate blend.

More accurate results can be obtained by using the carving knife to place the body and a strip of white blotting paper to absorb any surplus moistness. The flow of any color into another, can be stopped at once by placing the blotting paper on the body of porcelain placed in the matrix.

The form should be built up and carved to represent the form of the dentin in relation to that of the enamel which is accomplished in from two to three bakes. In the first bake a cross should be cut with the point of the carving spatula, to overcome the shrinkage and the bakes should be only brought to a high biscuit which gives an opaque translucency to the transmission of light, representing in all color, form and translucency as near as possible to that of dentin. The contour of the inlay is now completed with one color, representing the enamel, known as the enamel coat, and it should be placed with a fine pointed camel's hair brush. This is brought to a high glaze which reflects all the underlying colors of the previous bakes. The color of the enamel is selected from the color of the incisal third of the tooth which is usually of a grey blue or green hue. There are often found streaks of brown, yellow and white on the surfaces of the enamel and also faint checks of varying length and depth that have absorbed discoloration. These discolorations can be very artistically reproduced on the surface of the completed in- lay by the use of enamel stains which add a very beautiful artistic result to the operation.

6th. The building of contour and tooth form requires that the operator have at his command a thoro knowledge of dental anatomy, so that an inlay can be made to represent the form of the surface of the tooth in detail that is to be reproduced, and also, that when the inlay is cemented to place, there will not be any overhang at the cavo-surface angle, which will only require the slightest of finishing to complete the operation.

7th. Removal of the matrix and cementation. Great care should be taken in removing the matrix so as not to fracture the thin edges of the inlay in the least, and I wish to state, if such an accident should happen, a new inlay should be made in all cases, as such an inlay is faulty and should not be cemented to place. The inlay is held firmly between the thumb and index finger of the left hand while with the matrix pliers, the matrix is gently pulled away from the edges of the inlay first turning it in the fingers as you go; never pull the matrix from the body of the inlay towards the edge, or a fracture is likely to result.

When it is loosened from all the edges, then last of all remove it from the body of the inlay. Embed the inlay in black wax, exposing the surface that adapts to the cavity wall, being careful to have all the cavo-surface angles covered so they will not be distorted in the etching. This then is etched with hydrofloric acid for only a few seconds, depending on the strength of the acid. When etched, place under a stream of running water till the acid is thoroly washed off. Remove from the wax and wash with chloroform to remove any particles of wax that may remain; then wash it with alcohol and dry with warm air.

Cementation is a very important step 
and figures greatly into the permanency of any porcelain operation.

In proximal surfaces, the white and light yellow cements should only be used, but in the gingival surfaces the deeper yellows and greys can be used to some advantage. There is no advantage to try to color any inlav by the use of some color of cement. The principle to carry out is, to use a cement that will not change the color of the tooth and it is sometimes necessary, in deep cavities of decay where the enamel is somewhat underminded in the incisal third, to use some dense color of cement or color it grey or blue, by incorporating some powder of enamel stain in the cement, and only use this stained cement at this point placing it first, and in the rest of the cavity, and on the inlay, should be placed the lighter. cement that would be originally used if the case were normal. A bone or an agate spatula should be used to avoid discoloration from the incorporation of any metal in the cement mix. The cement should be mixed slowly and well spatulated, incorporating just a little powder at a time till the mix becomes syrupy; this is what is called a satisfied mix. The test for this is to raise the spatula from the slab, and in so doing it strings out like syrup. The mix is then complete and should be used as soon as possible. Therefore, preparation should be made so that as little time will be consumed in making the cementation as possible. In proximal surface operations, the inlay is drawn to place with a ligature, then a second ligature that has been well waxed; is passed around the tooth and tied with a double surgeon's knot drawing the inlay snugly to place. Care should be taken in not drawing this ligature too tight and fracturing the thin edges of the inlay at some point.

In gingival surfaces the inlay is rocked to place by the use of two wooden points, also using great care not to cause any fracture.
All freshly cemented inlays should be coated with varnish and the patient instructed not to disturb them for at least twelve hours, when the patient should return and the ligature removed and the operation finished. This consists of a very simple operation, when the inlay is made properly, and requires only to remove the overhang surplus cement. A fine cuttle fish strip is drawn over the gingival cavo-surface angle a few times and a cuttle fish disk in the engine is used to go over the lingual labial and incisal cavo surface angles, surfacing down the edge of the inlay to the smooth surface of the tooth, thus giving a clean and finished operation.

In addition to the limitations as outlined, I wish to state that there are conditions under which the use of the porcelain inlay is contra-indicated. A few principal ones are: Cases where the teeth are worn considerably from excessive use, especially in the proximoincisal surfaces of the incisors and cuspids. In badly discolored pulpless teeth, it would be nearly impossible to get a good shade. If caries has not been too extensive, a neatly outlined gold foil operation would be much more pleasing to the eye, or where the caries has been extensive, a jacket crown would be recommendable. And in the mouths of individuals who have a tendency to the green stain, the cement around the inlay becomes discolored, thus making . the operation very conspicuous.

In presenting this subject, time will not permit me to give you the detailed technic that is required in making porcelain inlay operations. Only the principles to be followed in a general way, have been considered. What I wish most to do than anything else, is to emphasize the fact that porcelain has a very important field in dentistry and the demand is going to fall on us to give this subject a broader and more conscientious consideration. 